\title{
Turning walking pneumonia into recurrent abscesses: a curious case of CVID and review of the literature
}

David X. Gao ( $\sim$ dgao7@uic.edu )

University of Illinois College of Medicine at Peoria https://orcid.org/0000-0002-1314-8800

Habiba Hussain

University of Illinois College of Medicine at Peoria

Brianna Bobber

University of Illinois College of Medicine at Peoria

Peter Phan

University of Illinois College of Medicine at Peoria

\section{Case report}

Keywords: common variable immunodeficiency, mycoplasma

Posted Date: February 26th, 2021

DOI: https://doi.org/10.21203/rs.3.rs-242505/v1

License: (a) (i) This work is licensed under a Creative Commons Attribution 4.0 International License.

Read Full License

Version of Record: A version of this preprint was published at Allergy, Asthma \&amp; Clinical Immunology on April 5th, 2022. See the published version at https://doi.org/10.1186/s13223-022-00673-3. 


\section{Abstract}

Background: common variable immunodeficiency (CVID) is a primary immunodeficiency disorder associated with a broad symptom presentation that is still being characterized. We report a rare case of recurrent mycoplasma skin abscesses in a patient with a history of autoimmune disorders and prolonged mycoplasma pneumonia who was diagnosed with CVID.

Case presentation: a 34-year-old woman presented with a history of recurrent abscesses previously confirmed positive for Mycoplasma pneumoniae. Her past medical history of recurrent mycoplasma abscesses, prolonged mycoplasma pneumonia, and autoimmune disorders (mixed connective tissue disease and immune thrombocytopenia) raised suspicion of CVID. Workup included negative antimycoplasma antibody titers, hypogammaglobulinemia, and negative anti-pneumococcal antibody titers despite prior vaccination, solidifying the diagnosis of CVID. The patient was discharged on antibiotic and intravenous immunoglobulin therapy with improvement and now follows allergy and immunology longterm for treatment.

Conclusions: her diagnostic history underscores the importance of considering the various criteria of CVID for diagnosis, and her unique presentation of M. pneumoniae skin abscesses highlights the broad sequelae patients with CVID can manifest.

\section{Background}

Common variable immunodeficiency (CVID) describes a syndrome of hypogammaglobinemia (immunoglobulin $\mathrm{G}[\mathrm{IgG}]$, immunoglobulin $\mathrm{A}[\mathrm{IgA}]+/$ - immunoglobulin $\mathrm{M}[\mathrm{IgM}]$ ) and impaired humoral response to antigens caused by failure of $B$ lymphocyte differentiation, in absence of other known immunodeficiency. Due to their deficient immune system, patients with CVID have an increased risk of recurrent respiratory infections, abscesses, pulmonary granulomas, inflammatory bowel disease, autoimmune conditions, and malignancy. ${ }^{1}$ However, there is no report on skin abscesses caused by mycoplasma. Herein, we report a case of mycoplasma skin abscesses in a patient whose past medical history raised suspicion for CVID. Her case provides a reminder of the broad diagnostic criteria and clinical sequelae patients with CVID manifest, and we aim to provide a useful summary of the diagnostic criteria, differential diagnosis, and treatment of CVID. We also provide information on this novel symptom of mycoplasma infection in patients with CVID, as well as in the general population.

\section{Case Presentation}

A 34-year-old woman with past medical history of mixed connective tissue disease (MCTD) previously on immunosuppressive therapy and immune thrombocytopenic purpura (ITP) status-post splenectomy presented with sepsis secondary to recurrent neck abscesses.

The patient reported two erythematous, painful, warm, and indurated areas on her chest and posterior neck that had been worsening over the past 10 days (Figure 1). She had a history of recurrent 
presentation of neck abscesses over the few years prior to this admission and most recently had a neck abscess drained one month prior. Bacterial culture from the drainage was noted to be negative, but Mycoplasma pneumoniae was detected upon microbial cell-free DNA analysis. She was started on oral azithromycin, which she had been taking daily until admission. No evidence of fever, dysphagia, or arthralgia in this time was noted.

In addition to ITP, past medical history revealed several years of recurrent abscesses of the neck and perianal region with negative cultures. In 2016 she had mycoplasma pneumonia lasting six months (Figure 2). She also was found to have pulmonary nodules which were biopsied, revealing granulomatous inflammation. Later that year she was diagnosed with MCTD for which she followed with rheumatology and was maintained on azathioprine and hydroxychloroquine (she self-discontinued both medications several months prior to admission out of concern for immunosuppression contributing to her recurrent abscesses). In 2018 she developed sepsis from lymphadenitis. A lymph node biopsy showed chronic inflammation with granulomatous features and serological detection of microbial cell-free DNA of $M$. pneumoniae. Warthin-Starry stain, Grocott-Gomori methanamine silver (GMS), and acid-fast bacilli stains (AFB) were negative.

Her vaccinations were up to date, including a pneumococcal conjugate vaccine (PCV-13) she had received following her splenectomy for ITP. She affirmed a strong family history of lupus and thyroid disease.

She initially met systemic inflammatory response syndrome (SIRS) criteria due to fever of $101.4^{\circ} \mathrm{F}$ and tachycardia. Physical exam revealed an atraumatic neck with diffuse cervical lymphadenopathy and three abscesses in the submental, supra-jugular, and right lateral posterior neck. She underwent incision and drainage and empiric treatment for M. pneumoniae with IV azithromycin. Her white blood cell count was within normal limits, but C-reactive protein was elevated at $5.86 \mathrm{mg} / \mathrm{dL}$ (range <.5). Gram stain, wound cultures, and blood cultures were negative. Broad range PCR was again positive for $M$. pneumonia.

Given her known history of recurrent and prolonged mycoplasma infections, her mycoplasma IgM and IgG antibodies were measured to rule out primary immunodeficiency. Both were revealed to be negative, raising concern for inability of memory B-cell differentiation. Further immunological workup revealed $\lg \mathrm{G}=541 \mathrm{mg} / \mathrm{dL}$ (range 552-631), $\lg A=83 \mathrm{mg} / \mathrm{dL}$ (range 65-421), and $\lg M=21 \mathrm{mg} / \mathrm{dL}$ (range 33-293); her hypogammaglobulinemia pointed toward possible underlying CVID. Her pneumococcal vaccine titers were measured, which were low for $11 / 23$ serotypes, solidifying the diagnosis of CVID. She subsequently received IVIG with improvement. Her remaining hospital course was unremarkable. She was discharged on oral azithromycin with a referral to allergy and immunology (A\&l) outpatient.

Shortly after discharge she returned to the hospital with a similar presentation, again underwent I\&D by surgery, received IVIG, and was prescribed levofloxacin. Immunoglobulin levels were followed, and IgG initially was within normal limits, but continued IVIG treatment was delayed due to insurance. IgG levels 
trended down and during this time, and her lymphadenopathy increased in size and number, for which doxycycline was added. IgA levels remained normal and IgM levels remained persistently low.

\section{Discussion}

CVID, as the second-most common primary immunodeficiency, presents with a range of manifestations. ${ }^{1}$ Skin sequelae are no exception: skin infections occur nearly four times as often in patients with CVID as compared to the general population, with diverse etiologies, including bacterial, viral, and fungal. Patients may also develop autoimmune diseases, such as vitiligo, alopecia areata, and psoriasis. ${ }^{2-4}$ Our case adds to this ever-expanding literature of unique presentations of CVID by being the first of our knowledge to describe M. pneumoniae skin abscess in a patient with CVID. We will first discuss the diagnosis and management of CVID, then the broad manifestations with which M. pneumoniae can present. Multiple diagnostic criteria for CVID exist, which we refer to here. ${ }^{5-7}$ Generally, these include: age greater than four, evidence of autoimmunity or recurrent bacterial infections, hypogammaglobulinemia (IgG and IgA and/or $\operatorname{lgM})$, poor antibody response to vaccinations, and no other identifiable cause for hypogammaglobulinemia. Moreover, involvement of multiple organ systems in CVID may lead to granulomatous inflammation, interstitial lung disease, autoimmune and hematologic disorders, and increased incidence of lymphocytic malignancy. ${ }^{8}$ With the exception of malignancy, our patient had all of the aforementioned criteria. Her history of recurrent infection with mycoplasma led us to suspect immunodeficiency and to test her mycoplasma antibody titers, which were negative. M. pneumoniae IgM and IgG should remain positive for several months during and after infection. ${ }^{9-10}$

With regards to the hypogammaglobulinemia criterion, IgG is traditionally at least two standard deviations below normal range in diagnoses of CVID. Another differential diagnosis for our patient included isolated IgG deficiency, which has a separate International Classification of Diseases code (D80.3) from CVID (83.9). An analysis by Filion et al. comparing two cohorts of patients with CVID and IgG deficiency found that, in addition to markedly low IgG, patients with CVID demonstrated significantly poorer vaccination response to the 23-valent pneumococcal polysaccharide vaccine (our patient was protected against $1 / 12$ serotypes in the Prevnar-13 vaccine) and higher rates of autoimmune disease (MCTD in our patient), autoimmune cytopenia (ITP), and granulomatous lesions (pulmonary granulomas). ${ }^{11}$ Although the immunoglobulin titers were higher than what one traditionally expects in a patient with CVID, our patient's comorbidities correlated to those more commonly found in CVID rather than IgG deficiency. This discrepancy attests to the necessity of thoroughly exploring the possible manifestations of CVID when differentiating between the two diagnoses.

The management of CVID consists of IVIG. ${ }^{12}$ Its mechanism in treating CVID has traditionally been thought of as replacement therapy, although recent evidence suggests that IVIG also induces proliferation and immunoglobulin synthesis in B cells, rectifying the defunct immunoglobulin synthesis pathway in these patients. ${ }^{13}$ In our patient, she reported improved wound healing and decreased malaise following her initial treatment in the hospital. Active infections may be treated with antibiotic therapy, as in our 
patient, wherein her persistent abscesses were treated with continued fluoroquinolone and tetracycline therapy. Patients should also avoid live vaccines and, due to their increased risk of malignancy, receive all age-appropriate cancer screenings.

With regards to M. pneumoniae infections, the most common location in CVID and non-CVID patients is in the respiratory tract. ${ }^{14-15}$ Rarer presentations of $M$. pneumoniae in the general population include central nervous system, cardiac, and cutaneous involvement. ${ }^{16-17}$

Several dermatologic manifestations of $M$. pneumoniae have been described. These include non-specific urticarial and maculopapular rashes, erythema multiforme, Stevens-Johnson syndrome, and $M$.

pneumoniaeinduced rash and mucositis. ${ }^{18}$ The mechanisms of these skin manifestations are thought to be via direct inoculation, secondary immune-mediated damage, vascular occlusion, or direct invasion of the vascular tissue, depending on the presentation. ${ }^{10,19}$ Some manifestations for example, such as the bullous lesions, are thought to be due to hematogenous transfer of the bacteria to the dermis, with damage caused by generation of inflammatory cytokines. ${ }^{10}$

Prior to this admission, our patient had previously demonstrated positivity of M. pneumoniae abscesses, implying direct invasion of the cutaneous tissue. Her negative culture during our admission points to secondary immune damage or hematogenous transfer from prior infections as the likely mechanism. Our patient had a normal white blood cell count throughout her admission, although leukopenia occurs in $25 \%$ of patients with CVID, making hematogenous transfer from one of her prior infections still a possible explanation for the positivity of her M. pneumoniae abscesses. ${ }^{20}$

\section{Conclusion}

In summary, this clinical vignette describes an unusual case of CVID manifesting with mycoplasma skin abscesses. Diagnosis was aided by her history of autoimmunity and recurrent infection, prompting suspicion of hypogammaglobulinemia and appropriate workup. This case serves as a reminder of the broad symptom manifestations of CVID and diverse diagnostic criteria clinicians should use to diagnose the syndrome. The important teaching point of this case is that, with such a variable presentation, CVID is an important diagnosis to keep on the differential for patients presenting with unexplained disease processes involving the aforementioned signs and symptoms.

\section{Abbreviations}

CVID: common variable immunodeficiency

MCTD: mixed connective tissue disease

ITP: immune thrombocytopenia

IVIG: intravenous immunoglobulin 
A\&l: allergy \& immunology

$\lg \mathrm{A}: \mathrm{A}$

IgE: immunoglobulin E

IgG: immunoglobulin G

GMS: Grocott-Gomori methanamine silver

AFB: acid-fast bacilli

PCV-13: pneumococcal conjugate

SIRS: systemic inflammatory response syndrome

IgM: immunoglobulin M

\section{Declarations}

\section{Ethics approval and consent to participate}

The patient consented to use of her data and her photographs for the case report.

\section{Consent for publication}

The patient consented to publication of the case report.

\section{Availability of data and materials}

Not applicable.

\section{Competing interests}

All authors declare that they have no competing interests.

\section{Funding}

Not applicable

\section{Author information and contributions}

\section{Author affiliations}

University of Illinois College of Medicine, Peoria, IL, USA.

David X. Gao, Habiba Hussain, Brianna Bobber, Peter Phan 


\section{Author contributions}

DXG drafted the manuscript. PP contributed to the conception of the work. All authors contributed to interpretation of the data, review of the electronic medical record, and critical revision of the work.

\section{Corresponding author}

Correspondence to David X. Gao.

\section{Acknowledgments}

We thank the American College of Physicians Downstate Illinois Chapter for the opportunity to present this work at their poster conference on November 12, 2019.

\section{References}

${ }^{1}$ Muşabak UH, Demirel F, Yeşillik S et al. Adults with common variable immunodeficiency: a single-center experience. Turk J Med Sci. 2017 Feb 27;47(1):1-12. doi: 10.3906/sag-1503-22.

${ }^{2}$ Shi $\mathrm{C}$, Wang $\mathrm{F}$, Tong A et al. NFKB2 mutation in common variable immunodeficiency and isolated adrenocorticotropic hormone deficiency: A case report and review of literature. Medicine (Baltimore). 2016 Oct;95(40):e5081. doi: 10.1097/MD.0000000000005081. Erratum in: Medicine (Baltimore). 2017 Nov 03;96(44):e8572.

${ }^{3}$ Gualdi G, Lougaris V, Baronio $\mathrm{M}$ et al. Burden of skin disease in selective IgA deficiency and common variable immunodeficiency. J Investig Allergol Clin Immunol. 2015;25:369-371.

${ }^{4}$ Miller LG, Eisenberg DF, Liu $\mathrm{H}$ et al. Incidence of skin and soft tissue infections in ambulatory and inpatient settings, 2005-2010. BMC Infect Dis. 2015;15:362. Published 2015 Aug 21. doi:10.1186/s12879015-1071-0

${ }^{5}$ Seidel MG, Kindle G, Gathmann B et al. The European Society for Immunodeficiencies (ESID) Registry Working Definitions for the Clinical Diagnosis of Inborn Errors of Immunity. J Allergy Clin Immunol Pract. 2019 Jul-Aug;7(6):1763-1770. doi: 10.1016/j.jaip.2019.02.004. Epub 2019 Feb 15.

${ }^{6}$ Ameratunga $\mathrm{R}$, Brewerton $\mathrm{M}$, Slade $\mathrm{C}$ et al. Comparison of diagnostic criteria for common variable immunodeficiency disorder. Front Immunol. 2014;5:415. Published 2014 Sep 15.

doi:10.3389/fimmu.2014.00415

${ }^{7}$ Bonilla FA, Barlan I, Chapel H et al. International Consensus Document (ICON): Common Variable Immunodeficiency Disorders. J Allergy Clin Immunol Pract. 2016 Jan-Feb;4(1):38-59. doi: 10.1016/j.jaip.2015.07.025. Epub 2015 Nov 7. 
${ }^{8}$ Cunningham-Rundles C. Common variable immune deficiency: case studies. Blood. 2019 Nov 21;134(21):1787-1795. doi: 10.1182/blood.2019002062.

${ }^{9}$ Sperlich JM, Grimbacher B, Workman S et al. Respiratory Infections and Antibiotic Usage in Common Variable Immunodeficiency. J Allergy Clin Immunol Pract. 2018;6(1):159-168.e3. doi:10.1016/j.jaip.2017.05.024

${ }^{10}$ Narita M. Pathogenesis of extrapulmonary manifestations of Mycoplasma pneumoniae infection with special reference to pneumonia. J Infect Chemother. 2010 Jun;16(3):162-9. doi: 10.1007/s10156-0100044-x. Epub 2010 Feb 27.

${ }^{11}$ Filion CA, Taylor-Black S, Maglione PJ, Radigan L, Cunningham-Rundles C. Differentiation of Common Variable Immunodeficiency From IgG Deficiency. J Allergy Clin Immunol Pract. 2019 Apr;7(4):1277-1284. doi: 10.1016/j.jaip.2018.12.004. Epub 2018 Dec 14.

${ }^{12}$ Paquin-Proulx D, Sandberg JK. Persistent Immune Activation in CVID and the Role of IVIg in Its Suppression. Front Immunol. 2014;5:637. Published 2014 Dec 16. doi:10.3389/fimmu.2014.00637

${ }^{13}$ Kaveri SV, Maddur MS, Hegde P, Lacroix-Desmazes S, Bayry J. Intravenous immunoglobulins in immunodeficiencies: more than mere replacement therapy. Clin Exp Immunol. 2011;164 Suppl 2(Suppl 2):2-5. doi:10.1111/j.1365-2249.2011.04387.

${ }^{14} \mathrm{Gelfand} \mathrm{EW}$. Unique susceptibility of patients with antibody deficiency to mycoplasma infection. Clin Infect Dis. 1993 Aug;17 Suppl 1:S250-3.

${ }^{15}$ Roifman CM, Rao CP, Lederman HM, Lavi S, Quinn P, Gelfand EW. Increased susceptibility to Mycoplasma infection in patients with hypogammaglobulinemia. Am J Med. 1986 Apr;80(4):590-4. doi: 10.1016/0002-9343(86)90812-0.

${ }^{16}$ Koskiniemi M, Rantalaiho T, Piiparinen $\mathrm{H}$ et al. Infections of the central nervous system of suspected viral origin: a collaborative study from Finland. J Neurovirol. 2001 Oct;7(5):400-8. doi: 10.1080/135502801753170255.

${ }^{17}$ Szymanski M, Petric M, Saunders FE, Tellier R. Mycoplasma pneumoniae pericarditis demonstrated by polymerase chain reaction and electron microscopy. Clin Infect Dis. 2002 Jan 1;34(1):E16-7. doi: 10.1086/338158. Epub 2001 Nov 27.

${ }^{18}$ Poddighe D, Bruni P. Mycoplasma pneumoniae-induced rash and mucositis (MIRM): an unusual mild skin rash associated with severe mucosal involvement. BMJ Case Rep. 2017 May 27;2017:bcr2017220749. doi: 10.1136/bcr-2017-220749.

${ }^{19}$ Bhoopalan SV, Chawla V, Hogan MB, Wilson NW, Das SU. Bullous Skin Manifestations of Mycoplasma pneumoniae Infection: A Case Series. J Investig Med High Impact Case Rep. 2017 Sep 
8;5(3):2324709617727759. doi: 10.1177/2324709617727759.

${ }^{20}$ Henson MR, Lugar PL. The Duke University Medical Center (DUMC) Experience with Common Variable Immune Deficiency (CVID). The Journal of Allergy and Clinical Immunology. 2013 Feb;131:AB72.

\section{Figures}
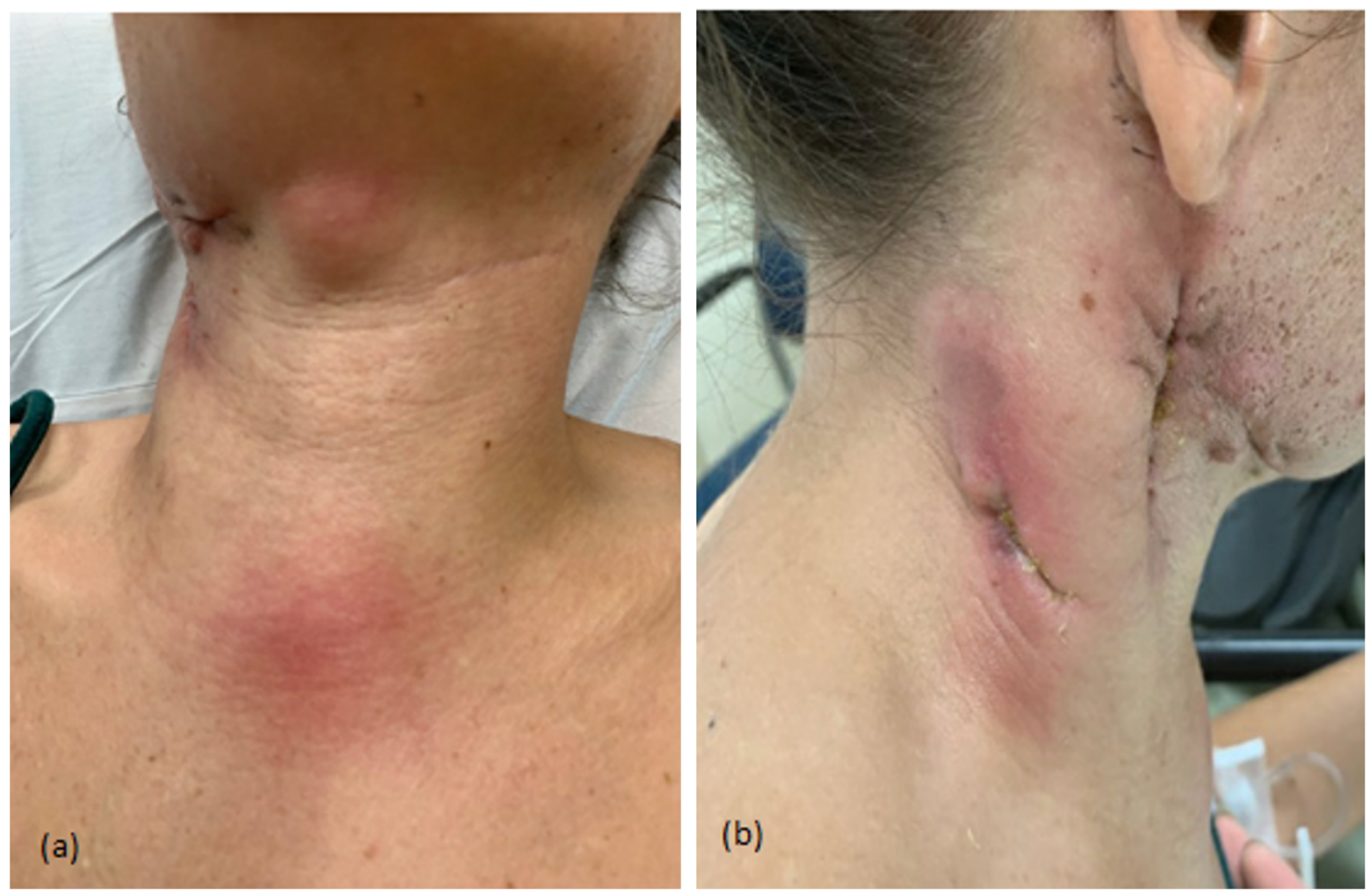

\section{Figure 1}

(left to right): (a) anterior neck abscess prior to incision and drainage. (b) Lateral neck abscess statuspost incision and drainage.

\begin{tabular}{|c|c|c|c|c|c|c|c|}
\hline Diagnosed with ITP, splenectomy & \multicolumn{2}{|c|}{ Work up for IBD due to perirectal abscesses } & \multicolumn{2}{|c|}{ Diagnosed with MCTD. Started Imuran and Plaquenil } & \multicolumn{2}{|c|}{$\begin{array}{l}\text { September 2018: } \\
\text { Large neck abscess I8D-Mycoplasma } \\
\text { pneumoniae }\end{array}$} & $\begin{array}{l}\text { August 2019: } \\
\text { Admitted for sepsis from neck abscess. } \\
\text { CVID work-up started }\end{array}$ \\
\hline \multicolumn{2}{|c|}{$\begin{array}{l}\text { 2011-2012: } \\
8 \text { ED visits/admissions for labial and perirectal abscesses }\end{array}$} & \multicolumn{2}{|c|}{$\begin{array}{l}\text { 2016: } \\
\text { Mycoplasma Pneumonia lasting } 6 \text { months. } \\
\text { Granulomas found on lung nodule biopsy }\end{array}$} & \multicolumn{2}{|c|}{$\begin{array}{l}\text { 2018: } \\
3 \text { admissions for sepsis from lymphadenitis }\end{array}$} & $\begin{array}{l}\text { Juna/July 2019: } \\
318 \mathrm{D} \text { of Neck abscesses }\end{array}$ & $\frac{\text { September 2019: }}{\text { First IVIG }}$ \\
\hline
\end{tabular}

\section{Figure 2}

timeline of patient's workup 